\title{
Stereotype Einstellungen und Kulturstandards in der aktuellen interkulturellen Kommunikation mit Partnern aus dem östlichen Europa - ein Forschungsprojekt am Institut für Interkulturelle Kommunikation
}

\author{
Roswitha Loew, Anke Pfeifer, Olga Rösch
}

\author{
Auf globalen Märkten agieren zu können, hängt von \\ der Kunst ab, anders zu denken. \\ (abgewandelt vom Werbeslogan des Beratungsunternehmens \\ Ernst \& Young in: Ost-West-Contact 1/2001)
}

\section{Ausgangspunkt}

In der Wirtschaftskommunikation zwischen Deutschland und den Staaten des ehemaligen Ostblocks ist das Wirtschaftsgefälle ein dominierender Faktor, der zu asymmetrischen Beziehungen führt. Diese manifestieren sich u. a. in Überlegenheits- und Unterlegenheitsgefühlen, die fuir die Ost-West-Kooperation eher kontraproduktiv sind. Außerdem wird die Kooperation mehr oder weniger durch eine gewisse Befangenheit, die aus dem geschichtlich „vorbelasteten“ Verhältnis (Zweiter Weltkrieg, Kalter Krieg) resultiert, begleitet.

Die unterschiedlichen Organisationskulturen, die aus der jeweiligen kulturellen Prägung erwachsen, erschweren eine optimale Zusammenarbeit gleichfalls, insbesondere zwischen den slawischen Staaten und Deutschland. Auch die Umstellung auf die Marktwirtschaft im ehemals sozialistischen Osten Europas erfolgt - in Bezug auf Tempo und Substanz - nicht immer nach den Wirtschaftskonzepten des Westens und führt damit nicht unbedingt zu der seitens Deutschland gewünschten Kompatibilität.

Angesichts der geographischen Lage der neuen Bundesländer und des einst intensiven Kontakts zu Ländern Osteuropas wurde von der ostdeutschen Wirtschaft nach der Wende sinnvollerweise erwartet, dass die Geschäftskontakte nach Osten ausgebaut werden. Die Entwicklung blieb jedoch hinter der Erwartung zurüick. Es wird z. Z. auch zunehmend beklagt, dass Unternehmen vor allem in den neuen Bundesländern zu wenig Wirtschaftskontakte zu den Ländern des östlichen Europas pflegen und dass das Interesse an Geschäften insbesondere mit Russland sogar nachlässt.

Die Entwicklung spiegelt sich auch in der Nachfrage nach Interkulturellen Trainings zu Osteuropa wider. Wenn man die Angebote an solchen Schulungen nach Ländern anschaut, dann stehen an erster Stelle bundesweit nach wie vor die USA. Ihnen folgt der ostasiatische Raum, vor allem China und Japan. Das Schlusslicht bilden die Länder Osteuropas [1]. Es entspricht keinesfalls der politi- schen und wirtschaftlichen Bedeutung der osteuropäischen Region für Deutschland, insbesondere angesichts der EU-Erweiterung Richtung Osten.

Nach Meinung von Fachleuten - sowohl der Wirtschaftsunternehmer als auch der Mediatoren (d. h. interkultureller Trainer) - gestalten sich die Geschäftskontakte zwischen deutschen und osteuropäischen Unternehmen besonders schwierig auf der kommunikativen Ebene. So schreiben Schroll-Machl/Lyskow-Strewe (2000:61-62) dazu:

„Interkulturelle Trainings zu Osteuropa stoßen ... auf mehr Probleme als Trainings zu anderen Ländern - vor allem auf der Ebene der Einstellungen. ... Stereotypisierungen gehören zum interkulturellen Alltag, doch das Ausmaß der Bereitschaft, sehr schnell und sehr rigide zu derartig massiven (Vor)Urteilen zu greifen, ist in Bezug auf (Ost)Mittel- und Osteuropa auffallend.“

Die Gründe sind sicherlich die eingangs genannten geschichtlichen Vorbelastungen, die bei aufgetretenen Schwierigkeiten Ressentiments wecken, wirtschaftliche Asymmetrie und die z. Z. ungünstigen Rahmenbedingungen (Rechtsunsicherheit, strukturelle Inkompatibilität), die das Interesse der deutschen Unternehmen an der Kooperation mit dem Osten dämpfen.

Die symmetrischen Beziehungen und eine optimale Kompatibilität bleiben ein durchaus anstrebenswertes Ideal. Realistisch gesehen wird es aber auch in der nahen Zukunft so bleiben wie es ist, was nicht bedeutet, dass hier keine positiven Entwicklungen stattfinden. Selbst wenn die osteuropäische Wirtschaft aufholt, werden die Rahmenbedingungen dort - nach unserem Dafürhalten - nie richtig „stimmen“. Sie bleiben „nicht deutsch“. Denn die sogenannten „Bedingungen“ sind nichts anderes als Ausdruck bzw. Ausprägung der jeweiligen Mentalität. Im Geschäftsleben gehören dazu Rechtsverständnis, Führungsstil, Machtstrukturen und Hierarchien, Problemlösungswege, Definition des Berufs- und Privatsphäre, Beziehungsgefuige, usw.

Die Geschäftskontakte mit ausländischen Partnern im Ausland gestalten sich grundsätzlich komplizierter als diese mit Landsleuten auf dem einheimischen Markt. Es gehört inzwischen zum Allgemeinwissen, dass der Erfolg eines im Ausland agierenden Unternehmens nicht allein von der Qualität und den Preisen seiner Produkte sowie deren Präsentation abhängt. Die Existenz unterschiedlicher kultureller Werte und Einstellungen bei beteilig- 
ten Interaktionspartnern wird bei wirtschaftlicher $\mathrm{Zu}$ sammenarbeit noch zu wenig beachtet. Daraus resultieren Schwierigkeiten bei Kooperationsbemühungen und Nachteile im Wettbewerb, die sich bei rechtzeitiger Berücksichtigung der sogenannten „soften“ Einflussfaktoren (spricht: interkulturelle Kompetenz der Mitarbeiter) durch die Unternehmensführung beseitigen ließen.

Die Verständigung zwischen Angehörigen verschiedener Kulturen erweist sich als eine Kernfrage in einer Zeit weltweiter politisch-ökonomischer, technischer und auch kultureller Vernetzung. Dabei geht es nicht ausschließlich und nicht in erster Linie um mangelnde Sprachkenntnisse, sondern um Probleme, die wesentlich komplexerer Natur sind. Diese können geschäftliche wie interpersonale Beziehungen nachhaltiger belasten als sprachliche Mängel.

Die Unterschätzung der Bedeutung von kulturellen Differenzen bei der Arbeit in bi- bzw. multikulturellen Projekten ist vor allem bei Unternehmen in den neuen Bundesländern zu beobachten. Die Gründe können sowohl materieller (Kosten für die Schulung der Mitarbeiter) als auch ideeller (Skepsis bezüglich des Nutzens von Interkulturellen Trainings) Art sein. Die jeweilige kulturelle Besonderheit der Arbeitsorganisation und des Kommunikationsstils, die zunächst unsichtbar zu sein scheinen, wird jedoch spätestens bei Misserfolgen in den negativen Bilanzen sichtbar werden.

Handlungs- und Leistungsorientierung der ausländischen Geschäftspartner, ihre Verhandlungsfuihrung, das Konfliktverhalten oder das Diskussionsverhalten, Umgang mit der Zeit, das Hierarchieverständnis oder die Auffassung uiber die Verbindlichkeit von Übereinkünften sind kulturspezifisch beeinflusst (Kulturstandards). Oft wird anderes Verhalten der Verhandlungspartner falsch ausgelegt; Konflikte und Missverständnisse sind somit vorprogrammiert. Fehlendes Verständnis für die Handlungsweisen des ausländischen Partners, aber auch Unwissenheit in Bezug auf die eigene kulturelle Prägung gefährden angebahnte Geschäftsbeziehungen, führen zu Konflikten innerhalb des Unternehmens oder lassen sie gänzlich scheitern. Allzu oft wird die fremdkulturelle Prägung gerade dann unterschätzt, wenn es sich um Partner aus benachbarten oder nahegelegenen Ländern handelt, also wenn vermutet wird, diese Kulturen seien der unseren ähnlich.

Gerade die neuen Bundesländer sind durch ihre geographische Lage dafür prädestiniert, der Forderung nach Intensivierung der Wirtschaftsbeziehungen zu den Ländern Ost-, Mittelost- und Südosteuropas nachzukommen. Eine stärkere Beachtung der Existenz unterschiedlicher Mentalitäten und Einstellungen bei Beteiligten, das Wecken von Verständnis für die Rolle kulturell bedingter Einflussfaktoren ist sowohl der Wirtschaftskooperation als auch der Unterstuitzung der Transformationsprozesse in den Ländern selbst höchst dienlich.

Die Kenntnis und die Berücksichtigung differenzierter kultureller Eigenarten (Kulturstandards), d. h. die Einbeziehung kulturellen Wissens und Handelns werden für die Herausbildung interkultureller Kompetenz und als Bedingung für erfolgreiche interkulturelle Wirtschaftskommunikation inzwischen zunehmend anerkannt. Den Prozessen interkultureller Kommunikation wird daher in Wissenschaft und Fortbildung weltweit verstärkt Aufmerksamkeit gewidmet.

\section{Zum Stand der Forschung}

Innerhalb der Kultur- und Sozialwissenschaften wird der Diskurs über kulturelle Differenzen und ihre Auswirkungen auf die interkulturelle Kommunikation seit einigen Jahren lebhaft geführt. Interkulturelle Kommunikation ist Kommunikation zwischen den Vertretern verschiedener Kulturen. Kultur wird von uns aufgefasst als Orientierungssystem, dass aus spezifischen Symbolen gebildet und in der jeweiligen Gesellschaft tradiert wird. Es beeinflusst das Wahrnehmen, Denken, Werten und Handeln aller ihrer Mitglieder und definiert somit deren Zugehörigkeit zur Gesellschaft. Kultur als Orientierungssystem strukturiert ein für die sich der Gesellschaft zugehörig fühlenden Individuen spezifisches Handlungsfeld und kulturspezifische Formen der Umweltbewältigung (Thomas, 1996, 112).

Diese spezifischen kulturellen Orientierungssysteme und Handlungsmuster werden mit dem Begriff des Kulturstandards erfasst, ein Arbeitsbegriff, mit dem kulturelle Determinanten im Interaktionsprozess beschreibbar und analysierbar gemacht werden können. Kulturstandards sind gesellschaftlich geprägt. Sie stehen im Zusammenhang mit Weltbildern, mit sozialen Normen und Handlungsmustern von Individuen. Kulturstandards formen Erklärungsmuster für Ursache- und Wirkungsabläufe, und sie prägen die Art und Weise, wie auf der Grundlage von Erfahrungen zielorientierte Handlungsstrategien für die Zukunft entworfen werden (Goodenough 1970, 1980, Götze 1987).

Auf der Grundlage des eigenkulturellen Orientierungssystems und der Kulturstandards wird insbesondere vom eigenen Verhalten Abweichendes wahrgenommen und beurteilt. Das kann zu Missverständnissen und Konflikten und in der Folge zu verstärkter Stereotypenbildung fuihren. Wird bei der Suche nach den Motiven oder Ursachen eines Missverständnisses oder eines Konfliktes auf eigene Wertvorstellungen oder Überzeugungen zurückgegriffen, ohne den fremdkulturellen Hintergrund zu berücksichtigen, ist das Nichtverstehen des anderen bzw. die Fehlinterpretation eines Ereignisses vorprogrammiert.

Die Nichterfuillung von Verhaltenserwartungen kann zu Störungen der Interaktion und gegebenenfalls zu Sanktionen gegenüber dem Interaktionspartner führen. Wir haben es hier mit interkulturellen Überschneidungssituationen zu tun, die eben überaus konfliktträchtig sind (Rothlauf, 1999). Die Aufdeckung, Beschreibung und Standardisierung von Kulturspezifik fördert Sicherheit im Umgang mit anderen, erzeugt Verständnis fuir ansonsten rätselhaft erscheinende Handlungen und hilft, Missverständnisse abzubauen. 
Wir gehen von der Annahme aus, dass Beschreibungen und Beurteilungen fremdkultureller Begegnungssituationen, in denen (kulturelle) Andersartigkeit der Interaktionspartner konstatiert wird, Fremdbilder reflektieren. Fremdbilder enthalten verschiedene inhaltliche Komponenten, zu denen neben Sachkenntnis oder Faktenwissen auch Deutungsschemata (Stereotypisierungen) gehören. Rückgriffe auf Fremdbilder konstituierende Komponenten wie einstellungsbezogene Stereotypisierungen werden immer dann geschehen, wenn kulturspezifische Kenntnisse fehlen bzw. unzureichend vorhanden sind. In diesem Falle sollen Deutungsschemata nach ihrem fremdkulturellen Hintergrund befragt werden, um nach historisch fundierten Bezügen zu Kulturstandards zu suchen. Dazu ist es erforderlich, die das Fremdbild konstituierenden explizit genannten Stereotype oder implizit vorhandene Stereotype zu ermitteln. (Ausfuihrlicher zum Thema Stereotypisierung des Fremden s. Rösch (Hrsg.), 2000).

Kulturstandards definieren wir uiber Alexander Thomas hinausgehend zwecks Abgrenzung zum Stereotyp als handlungsbezogene Konstrukte, deren Träger die jeweilige Fremdgruppe ist, in unserem Fall Ost- bzw. Südosteuropäer, und die reales durchschnittliches Verhalten in einer bestimmten Situation standardisiert definieren und deren Kenntnis und Berïcksichtigung einem fremdkulturellen Partner eine erfolgreiche interkulturelle Kommunikation ermöglicht. Stereotype betrachten wir als einstellungsbezogene Konstrukte, deren Träger die Eigengruppe ist, in unserem Fall Deutsche, und die anerkanntermaßen eine notwendige Orientierungs- und Ordnungsfunktion innehaben. Die bestehende Erwartungshaltung verhindert aber eher den produktiven Umgang mit Kulturstandards.

\section{Forschungsdefizite und Beitrag des Projektes zu deren Beseitigung}

Die Internationalisierung der Wirtschaft stellt neue Anforderungen an Kenntnisse und Fähigkeiten der Beteiligten, die nicht nur uiber spezielles Fachwissen, sondern gerade auch über Kompetenzen im Bereich der interkulturellen Kommunikation verfügen muissen. Es fehlt noch an Sensibilisierung, dass in der Begegnung von kulturell unterschiedlich geprägten Partnern, so auch bei Wirtschaftsoder Handelstätigkeit usw., immer Kulturtransfer stattfindet. Die fremdkulturelle Prägung wird insbesondere dann unterschätzt, wenn es sich um Partner aus Nachbarländern handelt, wie zum Beispiel Polen. Die Anerkennung der Anderen in Europa aber setzt die Anerkennung der Differenz voraus. Defizite auf diesem Wissensgebiet werden in wissenschaftlichen Publikationen immer wieder hervorgehoben (vgl. z. B. Ryssaev; Bolten/Dathe, 1995; Kappel, 1999). Die Fachpresse fordert dringend deren Beseitigung und beispielsweise die Aufnahme entsprechender Bildungsinhalte schon in das Ingenieurstudium.

Kulturstandards anderer Kulturen, die für Begegnungen Deutscher mit den entsprechenden Vertretern wichtig sind, wurden bisher lediglich für den amerikanischen und asiatischen Raum erfasst (USA - Müller/Thomas 1991, Markowsky/Thomas 1995; China - Thomas/Schenk, 1996; USA - Feichtinger, 1998). Hofstede, dessen Untersuchungen als ein Meilenstein in der kulturvergleichenden Forschung gilt, hat z. B. in seinen empirischen Erhebungen zur Bestimmung von Kulturen 50 Länder einbezogen. Er erhebt Anspruch auf universelle Gültigkeit seiner Ergebnisse, ohne dass Länder Ost-, Ostmittel- und Südosteuropas in diesen Untersuchungen beruicksichtigt wurden. Es fehlen in seinen aus quantitativ erhobenen Daten gewonnenen Ergebnissen also wesentliche Teile der Weltkultur.

Es können allerdings - eine kritische Sicht vorausgesetzt - eine Reihe von Erkenntnissen aus der Fremdbildforschung herangezogen werden, die aus mentalitätsgeschichtlichen und kulturvergleichenden Untersuchungen zu einzelnen Kulturen Osteuropas vorliegen. Des Weiteren sind in den vergangenen Jahren insbesondere in der interkulturellen Managementforschung Untersuchungen zu Transformationsprozessen in einzelnen Reformstaaten durchgefuihrt worden, in denen zumeist ausschnittweise spezielle Kommunikationsbereiche wie die Wirtschaftskommunikation mit relevanten innerbetrieblichen Problemen im Vordergrund stehen. Diese vorwiegend empirischen Analysen u. a. über arbeitsbezogene Wertorientierungen, über die Kulturspezifik des Führungshandelns, über Unternehmenskultur, Unterschiede der Ideensysteme in Markt- und Planwirtschaft etc. könnten Bausteine zur Identifizierung von Kulturstandards liefern bzw. zur Verifizierung von uns ermittelter Kulturstandards dienen. Dementsprechend werden diese Untersuchungen für unser Vorhaben Berücksichtigung finden (Bolten/Dathe 1995, Danne 1996, Lang 1996, Holtbrügge 1996, Lieberum, 1998, Rothlauf 1999, Catana/Catana 1999 u. a.)

Es kann also festgestellt werden, dass umfassende Untersuchungen zu Kulturstandards für den ost- und südosteuropäischen Raum bisher insgesamt fehlen, für Länder, die als aktuelle und potenzielle EU-Beitrittskandidaten zunehmend Partner in interkulturellen Kommunikationsbeziehungen sein werden. Hier besteht also eine empfindliche Forschungslücke, die wir mit diesem Projekt schließen wollen. Wir stellen uns nun die Aufgabe, aus kulturwissenschaftlicher Perspektive zentrale handlungswirksame Kulturstandards des ost- und südosteuropäischen Raumes, wie sie in der Interaktion mit Deutschen von Bedeutung sind, zu ermitteln, zu beschreiben und ihre kulturhistorischen Wurzeln aufzudecken.

Die Analyse wird geographisch eingegrenzt auf die Länder Polen, Ungarn, Russland und Rumänien, so dass jeweils ein Land des ostmittel-, mittel-, ost- und südosteuropäisches Raumes, das paradigmatisch für eine spezifische kulturhistorische Entwicklung steht, vertreten ist. Dabei sind folgende Hypothesen für uns forschungsleitend:

1. Ausgegangen wird von der Existenz kultureller Unterschiede, von unterschiedlichen Kulturstandards, und zwar exemplarisch in der russischen, polnischen, ungarischen und rumänischen Kultur im Vergleich zur deutschen Kultur. 
2. Kulturelle Unterschiede, die sich in Kulturstandards fassen lassen, werden in Berichten, Gesprächen, Erzählungen, die Begegnungen mit dem Anderskulturellen thematisieren, angesprochen.

3. Fremdkulturell bedingte konflikthafte Ereignisse werden häufig über tradierte stereotype Einstellungen und Vorurteile reflektiert, die dem Alltagsdiskurs entstammen. Stereotype Einstellungen haben einen Bezug zu fremdkulturellen Kulturstandards.

4. Kulturstandards sind kulturhistorisch determiniert, aber auch gesellschaftsabhängig und damit zeitlich einem gewissen Wandel unterworfen. Im Falle von Transformationsgesellschaften sind sie damit möglicherweise sogar generationsabhängig.

5. In Transformationsgesellschaften erfolgt in Bezug auf Kulturstandards einerseits eine Rückbesinnung auf traditionelle Werte sowie andererseits eine Anpassung an vermutete westliche Werte.

Der interdisziplinäre Ansatz des Forschungsprojektes erfordert den Einsatz einer Methodenkombination, die hier erstmals erprobt werden soll:

a) empirische Erhebungen zwecks Gewinnung von Texten (leitfadengestützte, strukturierte Interviews), Direkterhebung, qualitative Einzelerhebungen mit Fallbeschreibung;

b) komparatistisch-imagologische Untersuchung, die textlinguistische Aspekte berücksichtigt, zur Identifizierung von einstellungsbezogenen Stereotypisierungen;

c) qualitativ-interpretative Auswertung der gewonnenen Texte zur Identifizierung von handlungswirksamen Kulturstandards.

Erstmals soll im Zusammenhang mit der Identifizierung von Kulturstandards untersucht werden, inwieweit sich in stereotypen Einstellungen Deutscher uiber das östliche/südöstliche Europa und seine Bewohner Bezüge zu typischen Ausprägungen von fremdkulturellen Kulturstandards herstellen lassen. Das erfordert, den soziokulturellen Kontext in der fremden Kultur, der zu derartigen stereotypen Bewertungen gefuihrt hat und aus dem sich gleichzeitig bestimmte Kulturstandards selektieren lassen, zu hinterfragen und zu interpretieren.

\section{Durchführung des Forschungsvorhabens}

Unter dem Aspekt der besonderen Lage des Landes Brandenburg, insbesondere zu Ost- und Mittelosteuropa, werden brandenburgische Unternehmen als Untersuchungsgegenstand und Zielgruppe gewählt. Wir gehen davon aus, dass sich in diesem neuen Bundesland - zum Teil unter Rückgriff auf frühere Beziehungen zu ehemaligen sozialistischen Ländern - gerade besonders intensive Kontakte zu ost- mittelost- und südosteuropäischen Partnern finden lassen müssten. Methodisch sollen dazu interviewgestuitzte Erhebungen bei Wirtschaftsunternehmen und caritativen Einrichtungen im Land Brandenburg als einer Grenzregion zwischen Ost- und Westeuropa durchgefuihrt werden.
Schwerpunkte der Untersuchung werden sein:

1. Differenzen im interkulturellen Kontakt, die aus der Konfrontation von Personen mit unterschiedlichem kulturellem Hintergrund resultieren;

2. nicht konflikthaft erfahrene positiv verlaufene Begegnungssituationen im Umgang mit fremdkulturellen Partner, die aber Informationen über wertmäßig-kulturelle Unterschiede liefern können;

3. wahrgenommenen Ähnlichkeiten in der interkulturellen Kommunikation. Sie können Einfluss auf die weitere Zusammenarbeit haben, weil sie positive interpersonale Einstellungen befördern können;

4. stereotype Einstellungen unserer deutschen Gesprächspartner zur jeweiligen anderen Kultur, die auf die Identifizierung von Kulturstandards hinlenken könnten;

5. Die individuell vorgebrachten Stereotypen werden mit tradierten Stereotypen des deutschen öffentlichen Diskurses anhand schriftlicher Diskursfragmente (z.B. Pressetexte) abgeglichen und auf ihre Allgemeingültigkeit geprüft.

6. die historische Verwurzelung der ermittelten Kulturstandards in der jeweiligen Kultur anhand von kulturhistorischen und mentalitätsgeschichtlichen Texten und Abhandlungen;

7. mögliche Gemeinsamkeiten der für einzelne Kulturen herausgearbeiteten Kulturstandards, die für das östliche Europa relevant sind.

Mit den Ergebnissen soll Anstoß für weitere Forschungen über Kulturstandards im östlichen Raum Europas gegeben werden. Unter diesen Aspekten steht das Projekt im übergreifenden Kontext der Transformationsforschung, die einen zukünftig aufzubauenden Forschungsschwerpunkt an der Europa-Universität darstellen wird. In Zusammenarbeit mit dem Forschungsprojekt „Konsolidierungsprozesse in postsozialistischen Transformationsgesellschaften: Sozio-kulturelle Erfahrungs-, Deutungs- und Verhaltensmuster sozialer Gruppen“ im Forschungsverbund „Interdisziplinäre Transformationsforschung“" am F.I.T./Europa-Universität Viadrina, zu dem auch Netzwerkpartner des Zentrums für Zeithistorische Forschung Potsdam gehören, können die empirisch gewonnenen Untersuchungsergebnisse für die Erarbeitung grundlagentheoretischer Erkenntnisse über Deutung und Verhalten genutzt werden.

Anknüpfungspunkte zum Thema Kulturstandards bieten Forschung und Lehre an der Fakultät für Kulturwissenschaften der Europa-Universität Viadrina mit dem Schwerpunkt „Identität und Fremdheit“, in dessen Rahmen zu den für unser Forschungsprojekt so wichtigen Themen wie osteuropäische Fremdbilder, Individualitätskonzepte und Tabuforschung gearbeitet wird.

Neben dem wissenschaftlichen Erkenntniszuwachs will das Forschungsprojekt zur Verbesserung interkultureller Kompetenz beitragen. Geplant ist die direkte und schnelle Einbindung der aus der Praxis gewonnenen, wissenschaftlichen Erkenntnisse in die praktische 
Informations-, Bildungs- und Beratungsarbeit bezüglich der Vorbereitung und erfolgreichen Durchfuihrung von Auslandsgeschäften mit Unternehmen des östlichen und südöstlichen Europas. Den einzelnen Unternehmen und Einrichtungen kommt damit das Ergebnis wissenschaftlicher Forschungsarbeit unmittelbar zugute.

Die Forschungsergebnisse dienen des Weiteren als Basis für die Ausarbeitung von Bildungsprogrammen für Manager in öffentlichen Einrichtungen und der Wirtschaft, insbesondere kleiner und mittlerer Unternehmen in Brandenburg. Auf diese Weise können wirtschaftliche und andere Kontakte zu Ost- Mittelost- und Südosteuropa befördert, die Wettbewerbsfähigkeit brandenburgischer Wirtschaftsunternehmen gestärkt und als ein Gesamtanliegen des Projektes Vorurteile und Fremdenfeindlichkeit abgebaut werden.

Bildungspolitisch leistet die Erforschung von Kulturstandards generell einen Beitrag zum praktischen Verständnis kultureller Identifikations- und Kommunikationsprozesse. Die Kenntnis von Kulturstandards erleichtert es, Angehörige fremder Kulturen besser zu verstehen, Kommunikationsprobleme zu mindern und, was ein ganz besonderes Anliegen des Projektes ist, über Synergieeffekte Fremdenfeindlichkeit vorzubeugen. Ebenso bietet die Kenntnis von Kulturstandards die Möglichkeit, „Krisenmanagement“ zu betreiben und in einer fremdkulturellen Umgebung mit dortigen Verhaltensweisen und Denkgewohnheiten zurechtzukommen.

Die Ergebnisse sind für jenen Personenkreis von Wichtigkeit, der im Rahmen von Beziehungen zu ausländischen Partnern (politischer, wirtschaftlicher o. ä. Art) mit interkulturellen Überschneidungssituationen konfrontiert wird. Des Weiteren sind sie nutzbar für die Vorbereitung von Auslandsaufenthalten (Studierende, Absolventen, Fachleute aus der Region usw.) im östlichen Raum Europas.

Das vorliegende Projekt, das dankenswerterweise auch von den brandenburgischen IHKs und dem VDI Berlin unterstützt wird, integriert sich mit seiner Themenstellung organisch in das Profil des Instituts für Interkulturelle Kommunikation der Technischen Fachhochschule Wildau und unterstützt in besonderem Maße die hier angebundene Workshopreihe „Interkulturelle Kommunikation mit ausländischen Partnern in Wirtschaft und Wissenschaft". Mit seiner Zielstellung soll es wissenschaftliche Erkenntnisse bezüglich der spezifischen Unternehmenskommunikation von Partnern verschiedener Kulturen gewinnen, die in Lehre und Fortbildung erfolgreich genutzt werden können und somit den Wissenstransfer zwischen Forschung und Praxis gewährleisten.

\section{Anmerkung}

[1] Bei dieser Aufstellung handelt es sich allerdings nicht um eine statistische Erhebung.

\section{Literatur}

Bolten, J./M. Dathe (Hrsg.) (1995): Transformation und Integration: Aktuelle Probleme und Perspektiven west-/osteuropäischer Wirtschaftsbeziehungen. Berlin.

Catana, Al./D. Catana (1999): Romanian Cultural Background and its Relevance for Cross-Cultural Management. In: JEEMS, 3, S. 252-258.

Danne, G. (1996): Die Rolle von Mentalität und arbeitsbezogenen Wertstrukturen in Transformationsgesellschaften. Eine vergleichende Studie über kulturelle Werte, Arbeitsmotivation und Arbeitszufriedenheit in ostdeutschen, polnischen und westdeutschen Unternehmen. Hamburg.

Feichtinger, C. (1998): Individuelle Wertorientierungen und Kulturstandards im Ausland. Theorie, Empirie und Anwendung bei der Auslandsentsendung von Managern. Frankfurt am Main.

Goodenough, W.-H. (1980): Description and Comparison in Cultural Anthropology. Cambridge.

Götze, D. (1987): Probleme der Akkulturation und Assimilation. In: Reimann, H./H. Reimann (Hrsg.): Gastarbeiter. Analyse und Perspektiven eines sozialen Problems. 2. völlig neubearb. Aufl., Opladen, S. 67-94.

Hofstede, G. (1980): Culture's Consequences. International Differences in Work-Related Valutes. Beverly Hills.

Hofstede, G. (1991): Culture and Organizations - Software of the Mind. Cambrigde.

Hofstede, G./M. H. Bond (1984): Hofstedes culture dimensions: An independent validation using Rokeach's value survey. In: Journal of Cross-Cultural Psychology, 15, S. 417-433.

Hofstede, G./M.H. Bond (1988): The confucius connection: From cultural roots to economic growth. In: Organizational Dynamics, 16/4, S. 5-21.

Holtbrügge, D. (1996): Unternehmenskulturelle Anpassungsprobleme in deutsch-russischen Joint Ventures. In: Journal for East European Management Studies. vol 1/1, 1996, S. 7-53.

Kappel, Guido (1999): Verhandeln mit Russen. Bericht über ein Forschungsprojekt. In: Bungarten, Theo (Hrsg.), Sprache und Kultur in der interkulturellen Marketingkommunikation. Beiträge zur Wirtschaftskommunikation, Bd. 11, S. 93-105.

Lang, Rainhart (Hrsg.) (1996): Wandel von Unternehmenskulturen in Ostdeutschland und Osteuropa. II. Chemnitzer Ostforum, 6.-9.3.1995, München und Mehring.

Lieberum, U. (1998): Die Zusammenarbeit von deutschen und polnischen Führungskräften: eine empirische Untersuchung von deutsch-polnischen Unternehmen in Polen. Berlin.

Markowsky, R./A. Thomas (1995): Studienhalber in Deutschland. Orientierungstraining für amerikanische Studenten. Heidelberg.

Müller, A./A. Thomas (1991): Interkulturelles Orientierungstraining für die USA. Übungsmaterial. Saarbrücken.

Rösch, Olga (Hrsg.) (2000): Stereotypisierung des Fremden. Auswirkungen in der Kommunikation. Wildauer Schriftenreihe Interkulturelle Kommunikation, Bd. 4. Verlag News \& Media, Berlin.

Rothlauf, J. (1999): Interkulturelles Management. Mit Beispielen aus Vietnam, China, Japan, Rußland und Saudi-Arabien. München, Wien (WiSo-Lehr- und Handbücher).

Ryssaev, Ilschat (1994): Managementtrainingsbedarf in der Gemeinschaft Unabhängiger Staaten (GUS). In: Interkulturelle Kommunikation und Interkulturelles Training. Problemanalysen und Problemlösungen. Materialien zum Internationalen Kulturaustausch. Bd. 33, Stuttgart, S. 73-75. 
Schroll-Machl, S./Lyskow-Strewe, V. A. (2000): Erfahrungen mit Interkulturellen Trainings zu (Ost)Mittel- und Osteuropa. In: Organisationsentwicklung 2000, Heft 2, S. 56-67.

Thomas, A. (1996): Psychologie interkulturellen Handelns. Göttingen, Bern, Toronto, Seattle.

Thomas, A./E. Schenk (1996): Handlungswirksamkeit zentraler Kulturstandards in der Interaktion zwischen Deutschen und Chinesen. Regensburg.

\section{Autorinnen}

Dr. phil. Roswitha Loew

Technische Fachhochschule Wildau

Institut für Interkulturelle Kommunikation

\section{Dr. phil. Anke Pfeifer}

Technische Fachhochschule Wildau

Institut für Interkulturelle Kommunikation

\section{Dr. phil. Olga Rösch}

Technische Fachhochschule Wildau Institut für Interkulturelle Kommunikation Tel. (0 33 75) 508-367

E-Mail: roesch@sprz.tfh-wildau.de 